\title{
El Escorial, Real Biblioteca del Monasterio de San Lorenzo, Y.III.21 (gr.291)
}

Il codice di mm $270 \times 142$ consiste di ff. 195 e raccoglie una serie di scritti matematicoastrologici. Si compone di due unità codicologiche: la I (ff. 1-115), riferibile al XIV secolo, vede impegnati nella copia Argiro e alcuni suoi collaboratori; la seconda è stata riferita al XVI secolo, poiché gli scoli di Pediasimo Poto all'opera di Cleomede ivi recati sono stati copiati da Giacomo Diassorino (de Andrés, 175).

Nella I unità si distinguono numerosi testi e numerose mani note: nei ff. 1r-2v, recanti la parte finale del De cyclis solis et lunae ad Andronicum di Argiro (f. 1rv) e alcuni vaticini astrologici (f. 2rv) si deve riconoscere <Filoteo di Selimbria>; i. ff. 3r-8v contengono l'autografo di Argiro delle Novae Tabulae; i ff. 10r-88v contengono le Tabulae manuales di Tolomeo copiate da Argiro; i ff. 90r-93v il De usu astrolabii di Niceforo Gregora vergato dall'Anonimo B; nei fogli 94r-101r il De cyclis solis et lunae ad Andronicum, vergato da un collaboratore anonimo di Filoteo di Selimbria [alla sua mano si devono riferire anche: f. 12rv del Marc. gr. Z. 309 (coll. 300); ff. 83r-86v del Vat. Reg. gr. 116; ff. 75v-76v, 102r-107v, 110r-119v, 396v-398v dell’Ups. gr. 28], corretto in margine e completato (f. 1rv) da Filoteo. La mano di quest'ultimo ritorna ai ff. 102r$104 \mathrm{v}$, con un elenco di pleniluni a partire dall'anno 1388. Infine, i ff. 105r-112v [Euclide Elementa I.1-14] sono stati vergati dall'Anonimo A.

\section{Bibliografia}

\section{Cataloghi}

de Andrés (1965) II, 174-176.

\section{Studi}

Acerbi (2016) 187. 